\title{
A Game-Theoretic Precoding for Secure Communication in MIMO Interference Channels
}

\author{
Boyang Huang $\mathbb{D}$, Yanjun Fang $\mathbb{D}$, and Zhengmin Kong $\mathbb{D}$ \\ College of Power and Mechanical Engineering, Wuhan University, Wuhan, Hubei 430072, China \\ Correspondence should be addressed to Yanjun Fang; yjfang@whu.edu.cn
}

Received 11 June 2018; Accepted 12 August 2018; Published 26 August 2018

Academic Editor: Krzysztof Kecik

Copyright (C) 2018 Boyang Huang et al. This is an open access article distributed under the Creative Commons Attribution License, which permits unrestricted use, distribution, and reproduction in any medium, provided the original work is properly cited.

\begin{abstract}
We investigate the problem of secure communication in multiple-input-multiple-output (MIMO) interference channels from the perspective of physical layer security (PLS). We focus on the design of joint transceiver, which aims at maximizing the difference of mean-squared errors (MSE) between legitimate users and the eavesdropper. To reduce the complexity, the problem is formulated as a noncooperative game, and an asynchronous algorithm is proposed to obtain the Nash equilibrium solution. In each iteration, the closed-form solution is obtained by using Karush-Kuhn-Tucker (KKT) conditions. Furthermore, the performance of the joint transceiver, including both the secrecy rate and the MSE, is evaluated by simulations. Experimental result shows that the proposed algorithm has fast convergence speed and the security performance in different scenarios is effectively improved.
\end{abstract}

\section{Introduction}

Interference channels are usually used to model multiuser scenarios, such as cognitive radio systems, ad-hoc wireless networks, and various forms of broadcast channels [1]. In interference networks, multiple transmitters simultaneously communicate with their corresponding receivers. In terms of each receiver, signals from nonpaired transmitters are regarded as interference. Meanwhile, multiple-input and multiple-output (MIMO) technique has been proposed to improve spectral efficiency as well as multiplex gain in wireless communication systems. By using multiple antennas, higher information rate can be obtained in MIMO interference networks. Nevertheless, system complexity and difficulty of coding are increased rapidly.

Although the study of interference channel has a long history, the investigations are mainly focused on dealing with the multiuser interference, and researches on secure communication are relatively fewer [2-5]. Due to the broadcast nature, signals from any transmitter are shared by all access receivers, which means interference channels are vulnerable to eavesdropping without effective protection. In the past, the traditional cryptography that widely applied in wireless scenario has obtained quiet nice security performance. However, with the rapid development of computing speed, once quantum computers are put into practical, traditional cryptography will be greatly challenged by the brute force of quantum computing. Therefore, physical layer security (PLS) technology, which is defined from the perspective of information theory, has been proposed in the physical layer to complement and enhance the confidentiality provided by other layers [6, 7]. By PLS encoding, the quality of the legitimate channels is improved while the eavesdropping channels is degraded. As a benefit, even if the encoding leaked, security can still be guaranteed because of the advantage of channel quality [8].

In MIMO networks equipped with multiple antennas, beamforming has been widely recognized as an effective approach for improving the PLS [9-13]. More specifically, a precoder at transmitting side and a decoder at receiving side are jointly designed, which are known as the joint transceiver. In PLS, the secure communication can be admitted if the information rate of legitimate transmitter-receiver link is greater than that of the transmitter-eavesdropper link. To this end, researches on the sum secrecy rate maximization (SSRM) problem are carried out for interference channels. The authors of [14-16] designed the transceivers in MIMO interference network with two transmitter-receiver pairs aiming at maximizing sum secrecy rate. However, the analysis was limited to the two-users MIMO interference channels. 
Because the expression of the secrecy rate is quite complicated, the complexity of SSRM problem is rapidly increased with the number of users. Therefore, several authors select mean-squared error (MSE) instead of secrecy rate as the suboptimal indicator of security performance to reduce complexity. For interference network with $K$ transmitterreceiver pairs, a transceiver design based on minimizing MSE was proposed in [12], and the optimization problem was divided into several subproblems which can be solved easier. But this solution takes no eavesdropper (Eve) into consideration. In [17], the MSE of the Eve was considered, and an iterative distributed algorithm was proposed to minimize sum MSE. The two algorithms mentioned above are aimed at the global optimal solution, and encoding matrices of all transmitter-receiver pairs are computed simultaneously in each iteration, which will greatly increase complexity and computational time. Therefore, the transceiver design in interference networks is still an outstanding issue. Motivated by this challenge, we use game theory to solve the minimizing MSE problem and an asynchronous algorithm, which has low complexity and fast convergence speed, is proposed to obtain the Nash equilibrium (NE) solution.

In this paper, we consider a MIMO interference network with $K$ transmitter-receiver pairs, and any receiver has the potential to be Eve. The $k$ th transmitter broadcasts signals in the whole network, which are only meaningful for the $k$ th receiver. Accordingly, the $k$ th receiver will receive signals from all the transmitters, which are regarded as noises except for signals sent by the $k$ th transmitter. Eve is assumed as one of the receivers of $K$ transmitter-receiver pairs and tries to wiretap signals from any non-pair transmitter. For secure communication, we did the following contributions: (1) we build the wiretap model of the interference network with $K$ transmitter-receiver pairs. And statistically imperfect CSI model is adopted to guarantee the absolute robustness. (2) We use MSE to denote the security performance, and the security problem in MIMO interference networks is formulated as an optimization problem for the purpose of maximizing MSE difference between legitimate transmitter-receiver pairs and Eve. In detail, by adopting linear minimum meansquared errors (MMSE) receiver, transmit precoders (TPC) are regarded as the independent variables of the optimal problem. (3) We model a noncooperative game among all legitimate transmitter-receiver pairs, and each legitimate transmitter-receiver pair tries to minimize its MSE with security constraint. After that, an asynchronous algorithm is proposed to obtain the NE solution of joint transceivers. Specifically, the nonconvex MSE expressions are transformed into convex forms by an one-to-one mapping and the closeformed solution is obtained in each iteration.

The rest of this paper is organized as follows: based on information-theoretic viewpoint, Section 2 analyses the MIMO interference network and build the wiretap channel model; then, Section 3 formulates a noncooperative game and proposes an asynchronous algorithm to obtain the NE solution; in Section 4, the optimal joint transceiver with the purpose of minimizing the MSE of the estimated data between the legitimate transmitter-receiver pairs is solved; in Section 5, the performance of the joint transceiver is tested by simulation experiments and a detailed analysis is also conducted; finally, conclusion of this paper is stated in Section 6.

Notation. The following notation is used in this paper. Uppercase and lowercase boldface lines denote matrices and vectors, respectively. The operators $(\cdot)^{*},(\cdot)^{H}, \mathbb{E}(\cdot)$, and $\operatorname{tr}(\cdot)$ are conjugate, Hermitian, expectation, and trace operators. The operator $(\cdot)^{+}$is defined as $(x)^{+}=\max (0, x) .\|\cdot\|_{F}$ denotes the Frobenius norm of a matrix. $\mathscr{C} \mathscr{N}(0,1)$ represents the complex circularly symmetric Gaussian distribution with zero mean and unit variance. $\mathbb{C}$ denotes the complex field.

\section{System Model}

2.1. Wiretap Model. Here we consider an interference network of $K$ legitimate transmitter-receiver pairs. Each receiver can be the potential Eve, and wiretaps signals from nonpaired transmitters. As shown in Figure 1, the $k$ th transmitter, the $k$ th receiver, and Eve are equipped with $N_{t, k}, N_{r, k}$, and $N_{e}$ antennas, respectively. The $k$ th transmitter broadcasts signals which are only meaningful for the $k$ th receiver. And the $k$ th receiver will receive the signals not only from the $k$ th transmitter but also from other transmitters, which causes the mutual interference. We assume that Eve is trying to wiretap data from the $k$ th transmitter, and the wiretap model can be built from the perspective of $k$ th receiver and the Eve as

$$
\begin{aligned}
& y_{k}=\sum_{l=1}^{K} \mathbf{H}_{l k} x_{l}+n_{k} \\
& y_{e}=\sum_{l=1}^{K} \mathbf{H}_{l e} x_{l}+n_{e}
\end{aligned}
$$

where $y_{k} \in \mathbb{C}^{N_{r, k} \times 1}$ and $y_{e} \in \mathbb{C}^{N_{e} \times 1}$ are the vectors of signals received by the $k$ th receiver and Eve, respectively, and $x_{l} \in \mathbb{C}^{N_{t, k} \times 1}$ is the vector of signals transmit by the $l$ th transmitter. $\mathbf{H}_{l k} \in \mathbb{C}^{N_{r, k} \times N_{t, k}}$ and $\mathbf{H}_{l e} \in \mathbb{C}^{N_{r, k} \times N_{t, k}}$ are the channel matrices of the $l$ th transmitter to the $k$ th receiver link and the $l$ th transmitter to Eve link, respectively, and $n_{k} \in \mathbb{C}^{N_{r, k} \times 1}$ and $n_{e} \in \mathbb{C}^{N_{e} \times 1}$ are the complex additive white Gaussian noise (AWGN) vectors received by the $k$ th receiver and Eve, respectively. And we have $n_{k} \sim \mathscr{C} \mathscr{N}\left(0, \delta_{k}^{2} \mathbf{I}\right)$ and $n_{e} \sim \mathscr{C} \mathscr{N}\left(0, \delta_{e}^{2} \mathbf{I}\right)$, where $\delta_{k}^{2}$ and $\delta_{e}^{2}$ are the covariances of noises.

We place a joint transceiver which is consist of a TPC and a receive decoder (RDC) in each transmitter-receiver pair. For the $k$ th transmitter, the transmitted signal vector can be rebuilt as

$$
x_{k}=\mathbf{T}_{k} s_{k}
$$

where $s_{k} \in \mathbb{C}^{N_{r, k} \times 1}$ is the data symbol vector and $\mathbb{E}\left\{s_{k} s_{k}{ }^{H}\right\}=\mathbf{I}$; $\mathbf{T}_{k} \in \mathbb{C}^{N_{r, k} \times N_{r, k}}$ is the TPC matrix with independent columns. 


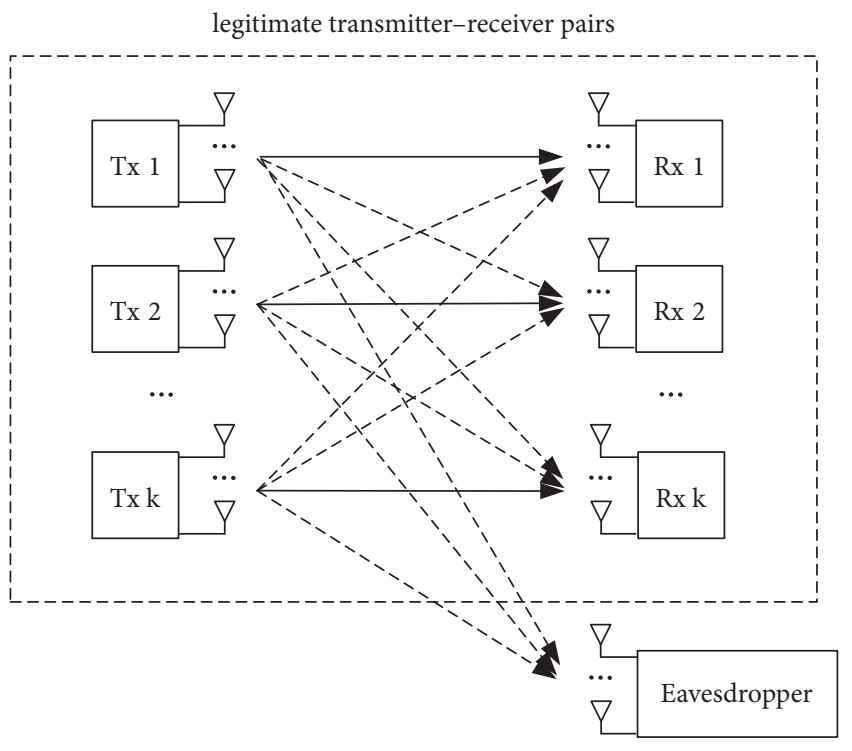

FIGURE 1: $K$-user MIMO interference channels with an eavesdropper.

Substituting (3) into (1) and (2), the received signals can be rewritten as

$$
\begin{aligned}
& y_{k}=\mathbf{H}_{k k} \mathbf{T}_{k} s_{k}+\sum_{l \neq k}^{K} \mathbf{H}_{l k} \mathbf{T}_{l} s_{l}+n_{k} \\
& y_{e}=\sum_{l=1}^{K} \mathbf{H}_{l e} \mathbf{T}_{l} s_{l}+n_{e}
\end{aligned}
$$

Let $\mathbf{R}_{k} \in \mathbb{C}^{N_{t, k} \times N_{r, k}}$ and $\mathbf{R}_{e, k} \in \mathbb{C}^{N_{t, k} \times N_{r, k}}$ denote the RDC matrices of the $k$ th receiver and Eve respectively, and the estimated data symbol vectors of the $k$ th receiver and Eve are given by

$$
\begin{gathered}
\widehat{s}_{k}=\mathbf{R}_{k}^{H} y_{k}=\mathbf{R}_{k}^{H} \mathbf{H}_{k k} \mathbf{T}_{k} s_{k}+\mathbf{R}_{k}^{H} \sum_{l \neq k}^{K} \mathbf{H}_{l k} \mathbf{T}_{l} s_{l}+\mathbf{R}_{k}^{H} n_{k} \\
\widehat{s}_{e, k}=\mathbf{R}_{e, k}^{H} y_{k}=\mathbf{R}_{e, k}^{H} \mathbf{H}_{k e} \mathbf{T}_{k} s_{k}+\mathbf{R}_{e, k}^{H} \sum_{l \neq k}^{K} \mathbf{H}_{l e} \mathbf{T}_{l} s_{l}+\mathbf{R}_{e, k}^{H} n_{e}
\end{gathered}
$$

In this paper, the linear MMSE receiver is selected as the $\mathrm{RDC}[18,19]$, which is formulated as

$$
\begin{aligned}
\mathbf{R}_{k} & =\left(\sum_{l=1}^{K} \mathbf{H}_{l k} \mathbf{T}_{l} \mathbf{T}_{l}^{H} \mathbf{H}_{l k}^{H}+\delta_{k}^{2} \mathbf{I}\right)^{-1} \mathbf{H}_{k k} \mathbf{T}_{k} \\
\mathbf{R}_{e, k} & =\left(\sum_{l=1}^{K} \mathbf{H}_{l e} \mathbf{T}_{l} \mathbf{T}_{l}^{H} \mathbf{H}_{l e}^{H}+\delta_{e}^{2} \mathbf{I}\right)^{-1} \mathbf{H}_{k e} \mathbf{T}_{k}
\end{aligned}
$$

As shown in (8) and (9), the RDC matrix depends on the set of TPC matrices $\left\{\mathbf{T}_{k}\right\}$. Therefore, we focus on the design of TPC matrices in the sections that follow.
2.2. Channel State Information. To reduce the multiuser interference, transmitter-receiver pairs share each other's CSI. In practice, training sequences are sent between each transmitter and receiver, and the estimation of CSI can be obtained at both transmitter and receiver side. In this paper, we consider the situation when Eve is not a hostile node, but one of the receivers in the alliance and the CSI of legitimate transmitter-receiver pairs is available to Eve as well. Meanwhile, although the feedback can facilitate the CSI estimation, there is always an error between the actual and estimated channel. To improve robustness, the statistically imperfect CSI model is adopted, and we have

$$
\begin{aligned}
\mathbf{H}_{l k} & =\overline{\mathbf{H}}_{l k}+\Delta \mathbf{H}_{l k} \\
\mathbf{H}_{l e} & =\overline{\mathbf{H}}_{l e}+\Delta \mathbf{H}_{l e}
\end{aligned}
$$

where $\overline{\mathbf{H}}_{l k}$ and $\overline{\mathbf{H}}_{l e}$ are the estimated channel of the $l$ th transmitter to $k$ th receiver and $l$ th transmitter to Eve links, respectively, while $\Delta \mathbf{H}_{l k}$ and $\Delta \mathbf{H}_{l e}$ denote the corresponding estimation error.

The uncertainty terms $\Delta \mathbf{H}_{l k}$ and $\Delta \mathbf{H}_{l e}$ are characterized by the widely adopted deterministic uncertainty model [20, 21]. In this model, $\Delta \mathbf{H}_{l k}$ and $\Delta \mathbf{H}_{l e}$ are bounded by the sets $\xi_{\mathbf{H}_{l k}}=\left\{\left\|\Delta \mathbf{H}_{l k}\right\|_{F} \leq \xi_{\mathbf{H}_{l k}}\right\}$ and $\xi_{\mathbf{H}_{l e}}=\left\{\left\|\Delta \mathbf{H}_{l e}\right\|_{F} \leq \xi_{\mathbf{H}_{l e}}\right\}$, respectively, where $\xi_{\mathbf{H}_{l k}}$ and $\xi_{\mathbf{H}_{l e}}$ are known constants. Relying on this deterministic model, we optimize the system's security performance under the worst-case channel condition. This approach guarantees the absolute robustness of the system design, since the achievable security performance is bounded to be no worse than the optimized result under the worst-case condition.

\section{Game-Theoretic Formulation}

In this section, the problem of TPC design is formulated as a noncooperative game and the NE solution is obtained by using an asynchronous algorithm. Different from algorithms that pursue global optimum, there is no need to calculate the TPC matrices of all transmitter-receiver pairs simultaneously. As a benefit, the arithmetic complexity is significantly reduced by solving the TPC matrix one by one.

3.1. Nash Equilibrium. The framework of a noncooperative game models a scenario where all legitimate transmitterreceiver pairs are regarded as competing players in a game. Each player acts independently and simultaneously according to their own interests with no a priori knowledge of other players' strategies. Specifically, we formulate the problem as follows.

Definition 1. Given a strategic form game $\mathscr{G}=$ $\left(\mathscr{K},\left\{Q_{k}\right\}_{k \in \mathscr{K}},\left\{\mathcal{U}_{k}\right\}_{k \in \mathscr{K}}\right)$, an action profile $\mathbf{T}^{*} \in \mathbb{Q}$ is a pure-strategy Nash equilibrium of $\mathscr{G}$ if the following condition holds for all $k \in \mathscr{K}$ :

$$
\mathcal{U}_{k}\left(\mathbf{T}_{k}^{*}, \mathbf{T}_{-k}^{*}\right) \geq \mathscr{U}_{k}\left(\mathbf{T}_{k}, \mathbf{T}_{-k}^{*}\right), \quad \forall \mathbf{T}_{k} \in Q_{k}
$$


where $\mathscr{K}=\{1,2, \ldots, K\}$ is the set of players; $Q_{k}$ is a nonempty set of the available pure strategies for the $k$ th player; $\mathscr{U}_{k}$ is the utility function of the $k$ th player.

The existence of NE is proved in Appendix A.

3.2. Asynchronous Algorithm. According to the NE analyzed above, an asynchronous algorithm, which is formally presented as Algorithm 1, is proposed to obtain the NE solution. The main procedure of our algorithm is given as follows:

(1) Firstly, the initialization of TPC matrices $\mathbf{T}_{k}$ is randomly generated and RDC matrices $\mathbf{R}_{k}$ and $\mathbf{R}_{e, k}$ are calculated in the sequel.

(2) Secondly, each TPC matrix $\mathbf{T}_{k}$ is updated according to the best response of the utility function based on TPC matrices of other players $\mathbf{T}_{-k}$ in the most recent time.

(3) Finally, the second step is repeated until utility functions of all players are converged. In other words, the Nash solution is obtained when none of the players will actively modify its TPC matrix.

\section{Optimal Joint Transceiver}

In this section, we solve the optimal joint transceiver by using the asynchronous algorithm mentioned in Section 3. Specifically, we first generate the utility function which is expressed by MSE. Then, a closed-form solution of the TPC matrix is deduced from the TPC matrix in last iteration.

4.1. Utility Function. In PLS, the secure rate is first proposed by Shannon and is defined as the difference of information rate between the legitimate user and Eve [22]. As shown in (13), if the secure rate is positive, the data symbol will be considered to be secure.

$$
R_{\text {secrecy }}=\left(R_{k}-R_{k, E v e}\right)^{+}
$$

where $R_{k}$ and $R_{k, E v e}$ are the information rate of the $k$ th transmitter to the $k$ th receiver link and the $k$ th transmitter to Eve link, respectively. In multiuser scenario, the communication rate is measured by signal-to-interference-plus-noise-ratio (SINR). Thus, $R_{k}$ and $R_{k, E v e}$ are given by

$$
\begin{aligned}
& R_{k}=\log \operatorname{det}(\mathbf{I} \\
& \left.+\frac{\mathbf{R}_{k}^{H} \mathbf{H}_{k k} \mathbf{T}_{k} \mathbf{T}_{k}^{H} \mathbf{H}_{k k}^{H} \mathbf{R}_{k}}{\sum_{l \neq k}^{K} \mathbf{R}_{k}^{H} \mathbf{H}_{l k} \mathbf{T}_{l} \mathbf{T}_{l}^{H} \mathbf{H}_{l k}^{H} \mathbf{R}_{k}+\delta_{k}^{2} \mathbf{R}_{k}^{H} \mathbf{R}_{k}}\right), \\
& R_{k, E v e}=\log \operatorname{det}(\mathbf{I} \\
& \left.\left.+\frac{\mathbf{R}_{e, k}^{H} \mathbf{H}_{k e} \mathbf{T}_{k} \mathbf{T}_{k}^{H} \mathbf{H}_{k e}^{H} \mathbf{R}_{e, k}}{\sum_{l \neq k}^{K} \mathbf{R}_{e, k}^{H} \mathbf{H}_{l e} \mathbf{T}_{l} \mathbf{T}_{l}^{H} \mathbf{H}_{l e}^{H} \mathbf{R}_{e, k}+\delta_{k}^{2} \mathbf{R}_{e, k}^{H} \mathbf{R}_{e, k}}\right), \ldots, K\right\} \\
& k \in\{1,2, \ldots, K\}
\end{aligned}
$$

However, differentiation of (14) and (15) has the shortcoming of computing expensive. Meanwhile, MSE is the suboptimal choice to reflect the communication quality. Therefore, we define the utility function as the difference of MSE between legitimate transmitter-receiver pair and Eve, which is formulated as

$$
\mathcal{U}_{k}\left(\mathbf{T}_{k}^{*}, \mathbf{T}_{-k}^{*}\right)=M S E_{\mathrm{Tx}_{k}, \mathrm{Eve}}-M S E_{\mathrm{Tx}_{k}, \mathrm{Rx}_{k}}
$$

where $M S E_{\mathrm{Tx}_{k}, \mathrm{Rx}_{k}}$ and $M S E_{\mathrm{Tx}_{k} \text {,Eve }}$ are the MSE of the $k$ th transmitter to the $k$ th receiver link and the $k$ th transmitter to Eve link, respectively. $M S E_{\mathrm{Tx}_{k}, \mathrm{Rx}_{k}}$ and $M S E_{\mathrm{Tx}_{k} \text {, Eve }}$ are given by

$$
\begin{aligned}
& M S E_{\mathrm{Tx}_{k}, \mathrm{Rx}_{k}}=\operatorname{tr}\left(\mathrm{MSE}_{\mathrm{Tx}_{k}, \mathrm{Rx}_{k}}\right) \\
& =\operatorname{tr}\left(\mathbb{E}\left\{\left(\widehat{s}_{k}-s_{k}\right)\left(\widehat{s}_{k}-s_{k}\right)^{H}\right\}\right) \\
& =\operatorname{tr}\left(\mathbf{R}_{k}^{H}\left(\sum_{l=1}^{K} \mathbf{H}_{l k} \mathbf{T}_{l} \mathbf{T}_{l}^{H} \mathbf{H}_{l k}^{H}\right) \mathbf{R}_{k}-\mathbf{R}_{k}^{H} \mathbf{H}_{k k} \mathbf{T}_{k}\right. \\
& \left.-\mathbf{T}_{k}^{H} \mathbf{H}_{k k}^{H} \mathbf{R}_{k}+\delta_{k}^{2} \mathbf{R}_{k}^{H} \mathbf{R}_{k}+\mathbf{I}\right) \\
& M S E_{\mathrm{Tx}_{k}, \text { Eve }}=\operatorname{tr}\left(\mathbf{M S E}_{\mathrm{Tx}_{k}, \text { Eve }}\right) \\
& =\operatorname{tr}\left(\mathbb{E}\left\{\left(\widehat{s}_{e, k}-s_{k}\right)\left(\widehat{s}_{e, k}-s_{k}\right)^{H}\right\}\right) \\
& =\operatorname{tr}\left(\mathbf{R}_{e, k}^{H}\left(\sum_{l=1}^{K} \mathbf{H}_{l e} \mathbf{T}_{l} \mathbf{T}_{l}^{H} \mathbf{H}_{l e}^{H}\right) \mathbf{R}_{e, k}-\mathbf{R}_{e, k}^{H} \mathbf{H}_{k e} \mathbf{T}_{k}\right. \\
& \left.-\mathbf{T}_{k}{ }^{H} \mathbf{H}_{k e}^{H} \mathbf{R}_{e, k}+\delta_{e}^{2} \mathbf{R}_{e, k}^{H} \mathbf{R}_{e, k}+\mathbf{I}\right)
\end{aligned}
$$

4.2. Solution of Optimal Joint Transceiver. For the sake of maximizing the utility function, there are two intuitive strategies. The first one is that setting the minimum threshold of MSE to an acceptable constant, any value greater than it will be considered as a representation of poor communication quality. Then, we minimize the MSE of the $k$ th transmitter to the $k$ th receiver link. On the contrary, the second one is maximizing the MSE of the $k$ th transmitter to Eve link while setting the MSE of the $k$ th transmitter to the $k$ th receiver link to an acceptable value. Here we adopt the first strategy which can be described as an optimization problem as follows:

$$
\begin{array}{ll} 
& \mathbf{T}_{k}^{*}=\arg \min _{\left\{\mathrm{T}_{k}\right\}} M S E_{\mathrm{Tx}_{k}, \mathrm{Rx}_{k}}, \\
\text { s.t. } & M S E_{\mathrm{Tx}_{k}, \text { Eve }} \geq \varepsilon_{k}, \quad \forall k \in\{1,2, \ldots, K\}, \\
& \left\|\mathbf{T}_{k}\right\|_{F}^{2} \leq p_{k}, \quad \forall k \in\{1,2, \ldots, K\}
\end{array}
$$

where $\mathbf{T}_{k}^{*}$ is the optimal solution obtained; $\varepsilon_{k}$ is the minimum threshold of MSE of the $k$ th transmitter to Eve link; $P_{k}$ is the maximum power constraint imposed on the $k$ th transmitter. In fact, enough transmitted power is necessary to keep $M S E_{\text {Eve,Tx }_{k}}$ bigger than $\varepsilon_{k}$. Therefore, the optimization problem can be simplified by ignoring the transmitted 
1. Initialization: set the iteration counter to $n=0$, and start with an arbitrary TPC matrix $\mathbf{T}_{k}^{(n)}=\mathbf{T}_{k}{ }^{(0)}$.

2. Begin the iteration: calculate and update $\mathbf{R}_{k}^{(n+1)}$ and

$\mathbf{R}_{e, k}^{(n+1)}$ according to the MMSE receivers in (8) and (9), respectively. 3. Update TPC matrix $\left\{\mathbf{T}_{k}^{(n+1)}\right\}$ :

for $k=1$ to $K$ do

end

$$
\left\{\mathbf{T}_{k}^{(n+1)}\right\}= \begin{cases}\arg \max \mathscr{U}_{i}\left(\mathbf{T}_{i}^{*}, \mathbf{T}_{-i}^{(n)}\right), & i=k \\ \mathbf{T}_{i}^{(n)}, & \text { otherwise }\end{cases}
$$

4. Repeat Step 2 through 3 until $\left\{\mathscr{U}_{i}\right\}$ is converged.

Algorithm 1: Asynchronous Algorithm.

power constraint and the mathematical proof is shown in Appendix B.

To start with, we transform the expression of RDC matrix in (8) as follows:

$$
\begin{aligned}
\mathbf{R}_{k} & =\left(\sum_{l=1}^{K} \mathbf{H}_{l k} \mathbf{T}_{l} \mathbf{T}_{l}^{H} \mathbf{H}_{l k}^{H}+\delta_{k}^{2} \mathbf{I}\right)^{-1} \mathbf{H}_{k k} \mathbf{T}_{k} \\
& =\left(\mathbf{H}_{k k} \mathbf{T}_{k} \mathbf{T}_{k}^{H} \mathbf{H}_{k k}^{H}+\sum_{l \neq k}^{K} \mathbf{H}_{l k} \mathbf{T}_{l} \mathbf{T}_{l}^{H} \mathbf{H}_{l k}^{H}+\delta_{k}^{2} \mathbf{I}\right)^{-1} \\
& \cdot \mathbf{H}_{k k} \mathbf{T}_{k}=\left(\mathbf{H}_{k k} \mathbf{T}_{k} \mathbf{T}_{k}^{H} \mathbf{H}_{k k}^{H}+\mathbf{\Phi}_{k}+\delta_{k}^{2} \mathbf{I}\right)^{-1} \mathbf{H}_{k k} \mathbf{T}_{k}
\end{aligned}
$$

where $\boldsymbol{\Phi}_{k}=\sum_{l \neq k}^{K} \mathbf{H}_{l k} \mathbf{T}_{l} \mathbf{T}_{l}^{H} \mathbf{H}_{l k}^{H}$. By substituting (20) into (17) and using the matrix inversion lemma, the MSE matrix of the $k$ th transmitter to the $k$ th receiver link is simplified as

$$
\begin{aligned}
\mathbf{M S E}_{\mathrm{Tx}_{k}, \mathrm{Rx}_{k}} & =\mathbf{I}+\mathbf{T}_{k}^{H} \mathbf{H}_{k k}^{H}\left(\boldsymbol{\Phi}_{k}+\delta_{k}^{2} \mathbf{I}\right)^{-1} \mathbf{H}_{k k} \mathbf{T}_{k} \\
& =\left(\mathbf{I}+\mathbf{T}_{k}^{H} \mathbf{H}_{k k}^{H} \mathbf{\Omega}_{k} \mathbf{H}_{k k} \mathbf{T}_{k}\right)^{-1}
\end{aligned}
$$

where $\boldsymbol{\Omega}=\left(\boldsymbol{\Phi}+\delta_{k}^{2} \mathbf{I}\right)^{-1}$. Similarly, the MSE matrix of the $k$ th transmitter to the $k$ th receiver link is as follows:

$$
\mathbf{M S E}_{\mathrm{Tx}_{k}, \text { Eve }}=\left(\mathbf{I}+\mathbf{T}_{k}^{H} \mathbf{H}_{k k}^{H} \boldsymbol{\Omega}_{e, k} \mathbf{H}_{k k} \mathbf{T}_{k}\right)^{-1}
$$

where $\boldsymbol{\Omega}_{e, k}=\left(\sum_{l \neq k}^{K} \mathbf{H}_{e, l} \mathbf{T}_{l} \mathbf{T}_{l}^{H} \mathbf{H}_{e, l}^{H}+\delta_{e, k}^{2} \mathbf{I}\right)^{-1}$. Then, the optimization problem in (17) can be transformed as

$$
\begin{aligned}
\mathbf{T}_{k}^{*}=\underset{\mathbf{T}_{k}}{\arg \min } \operatorname{tr}\left(\mathbf{I}+\mathbf{T}_{k}^{H} \mathbf{H}_{k k}^{H} \mathbf{\Omega}_{k} \mathbf{H}_{k k} \mathbf{T}_{k}\right)^{-1} \\
\text { s.t. } \quad \operatorname{tr}\left(\mathbf{I}+\mathbf{T}_{k}^{H} \mathbf{H}_{k k}^{H} \boldsymbol{\Omega}_{e, k} \mathbf{H}_{k k} \mathbf{T}_{k}\right)^{-1} \geq \varepsilon_{k}, \\
\forall k \in\{1,2, \ldots, K\}
\end{aligned}
$$

To facilitate the analysis, we use the fact that $\mathbf{H}_{k k}^{H} \boldsymbol{\Omega}_{k} \mathbf{H}_{k k}$ and $\mathbf{H}_{k k}^{H} \boldsymbol{\Omega}_{e, k} \mathbf{H}_{k k}$ are Hermitian matrices and have the same rank. Therefore, we can use a nonsingular matrix $\mathbf{C}$ to diagonalize $\mathbf{H}_{k k}^{H} \boldsymbol{\Omega}_{k} \mathbf{H}_{k k}$ and $\mathbf{H}_{k k}^{H} \boldsymbol{\Omega}_{e, k} \mathbf{H}_{k k}$ simultaneously [23].

$$
\begin{gathered}
\mathbf{C}^{H} \mathbf{H}_{k k}^{H} \boldsymbol{\Omega}_{k} \mathbf{H}_{k k} \mathbf{C}=\boldsymbol{\Lambda}_{b}=\operatorname{diag}\left(\lambda_{b, i}\right) \\
\mathbf{C}^{H} \mathbf{H}_{k k}^{H} \boldsymbol{\Omega}_{e, k} \mathbf{H}_{k k} \mathbf{C}=\boldsymbol{\Lambda}_{e}=\operatorname{diag}\left(\lambda_{e, i}\right)
\end{gathered}
$$

where $\lambda_{b, i} \geq 0, \forall i \in\{1,2, \ldots, N\}$ and $\lambda_{e, i} \geq 0, \forall i \in$ $\{1,2, \ldots, N\} ; N$ is the dimensions of the diagonal matrix. In [24], the optimal TPC matrix in MIMO wiretap channel is proved to follow the diagonal structure which is given by

$$
\mathbf{T}_{k}=\operatorname{Cdiag}\left(\sqrt{\sigma_{i}}\right)
$$

where $\sigma_{i} \geq 0, \forall i \in\{1,2, \ldots, N\}$. The problem (23) can be simplified as

$$
\begin{array}{cl} 
& \mathbf{T}_{k}^{*}=\underset{\mathbf{T}_{k}}{\arg \min } \sum_{i=1}^{N}\left(1+\lambda_{b, i} \sigma_{i}\right)^{-1} \\
\text { s.t. } & \sum_{i=1}^{N}\left(1+\lambda_{e, i} \sigma_{i}\right)^{-1} \geq \varepsilon_{k}, \quad \forall k \in\{1,2, \ldots, K\}
\end{array}
$$

However, the objective function in (26) is monotone and nonconvex. Thus, we transform the objective function to a standard convex function by adopting the one-to-one mapping $\sigma_{i}^{\prime}=\left(1+\lambda_{e, i} \sigma_{i}\right)^{-1}$ :

$$
\begin{array}{ll} 
& \mathbf{T}_{k}^{*}=\underset{\mathbf{T}_{k}}{\arg \min } \sum_{i=1}^{N} \frac{\sigma_{i}^{\prime}}{\sigma_{i}^{\prime}\left(1-\lambda_{b, i} / \lambda_{e, i}\right)+\lambda_{b, i} / \lambda_{e, i}} \\
\text { s.t. } & \sigma_{i}^{\prime} \geq \varepsilon_{k}, \quad \forall k \in\{1,2, \ldots, K\} \\
& 0 \leq \sigma_{i}^{\prime} \leq 1
\end{array}
$$

The Lagrangian function of the optimization problem in (27) is given by

$$
\begin{aligned}
L\left(\mathbf{T}_{k}, \mathbf{R}_{k}, \mu_{k}, \lambda_{k}\right)= & \sum_{i=1}^{N} \frac{\sigma_{i}^{\prime}}{\sigma_{i}^{\prime}\left(1-\lambda_{b, i} / \lambda_{e, i}\right)+\lambda_{b, i} / \lambda_{e, i}} \\
& +\lambda_{k}\left[\varepsilon_{k}-\sigma_{i}^{\prime}\right]+\mu_{k}\left(0-\sigma_{i}^{\prime}\right) \\
& +\mu_{k}^{\prime}\left(\sigma_{i}^{\prime}-1\right)
\end{aligned}
$$




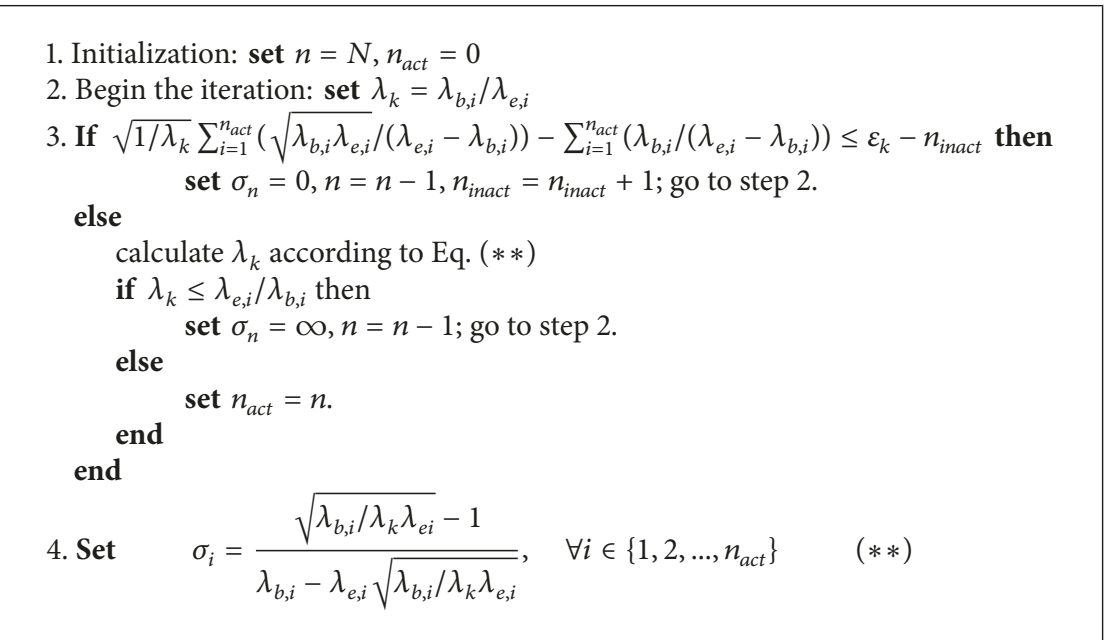

Algorithm 2: Algorithm to solve TPC matrix.

where $\lambda_{k}, \mu_{k}$, and $\mu_{k}^{\prime}$ are the Lagrange multipliers. In order to obtain the optimal solution, the following Karush-KuhnTucker (KKT) conditions have to be satisfied:

$$
\begin{aligned}
\frac{\partial L\left(\mathbf{T}_{k}, \mathbf{R}_{k}, \mu_{k}, \lambda_{k}\right)}{\partial \sigma_{i}^{\prime}} & =0 \Longrightarrow \\
\frac{\lambda_{b i} / \lambda_{e i}}{\left[\sigma_{i}^{\prime}\left(1-\lambda_{b i} / \lambda_{e i}\right)+\lambda_{b i} / \lambda_{e i}\right]^{2}} & =\lambda_{k}+\mu_{k}-\mu_{k}^{\prime} \\
\lambda_{k}\left[\varepsilon_{k}-\sigma_{i}^{\prime}\right] & =0, \quad \lambda_{k} \geq 0 \\
\mu_{k}\left(0-\sigma_{i}^{\prime}\right) & =0, \quad \mu_{k} \geq 0 \\
\mu_{k}^{\prime}\left(\sigma_{i}^{\prime}-1\right) & =0, \quad \mu_{k}^{\prime} \geq 0
\end{aligned}
$$

Combining (29), (30), (31), and (32), the optimal solution is given by

$$
\begin{aligned}
& \sigma_{i}^{\prime}=1, \quad \frac{\lambda_{b, i}}{\lambda_{e, i}} \leq \lambda_{k} \\
& \sigma_{i}^{\prime}=\frac{\sqrt{\lambda_{b, i} \lambda_{e, i} / \lambda_{k}}-\lambda_{b, i}}{\lambda_{e, i}-\lambda_{b, i}}, \quad \frac{\lambda_{e, i}}{\lambda_{b i}} \leq \lambda_{k} \leq \frac{\lambda_{b, i}}{\lambda_{e i}} \\
& \sigma_{i}^{\prime}=0, \quad \frac{\lambda_{e, i}}{\lambda_{b, i}} \geq \lambda_{k}
\end{aligned}
$$

Consequently, the allocation of $\sigma_{i}$ can be derivable from

$$
\begin{aligned}
& \sigma_{i}=0, \quad \frac{\lambda_{b, i}}{\lambda_{e, i}} \leq \lambda_{k} \\
& \sigma_{i}=\frac{\sqrt{\lambda_{b, i} / \lambda_{k} \lambda_{e, i}}-1}{\lambda_{b, i}-\lambda_{e, i} \sqrt{\lambda_{b, i} / \lambda \lambda_{e, i}}}, \quad \frac{\lambda_{e, i}}{\lambda_{b, i}} \leq \lambda_{k} \leq \frac{\lambda_{b, i}}{\lambda_{e, i}} \\
& \sigma_{i}=\infty, \quad \frac{\lambda_{e, i}}{\lambda_{b, i}} \geq \lambda_{k}
\end{aligned}
$$

Obviously, (34) is a piecewise function which is segmented by $\lambda_{k}$. And $\lambda_{k}$ can be derived by substituting (33) into (30):

$$
\sqrt{\lambda_{k}}=\frac{\sum_{i=1}^{n_{a c t}}\left(\sqrt{\lambda_{b i} \lambda_{e i}} /\left(\lambda_{e i}-\lambda_{b i}\right)\right)}{\sum_{i=1}^{n_{a c t}}\left(\lambda_{b i} /\left(\lambda_{e i}-\lambda_{b i}\right)\right)+\varepsilon_{k}-n_{\text {inact }}}
$$

where $n_{\text {act }}$ and $n_{\text {inact }}$ are the numbers of active data streams (where $0<\sigma_{i}<\infty$ ) and inactive data streams (where $\sigma_{i}=0$ ), respectively.

By observing (34) and (35), we find that $\sigma_{i}$ and $\lambda_{k}$ depend on each other and if $n_{\text {inact }}$ is known, both $\sigma_{i}$ and $\lambda_{k}$ can be solved. Therefore, we propose a searching algorithm to obtain $n_{\text {inact }}$ by comparing $\lambda_{k}$ and boundary conditions in (34). In detail, we first rearrange the values of $\lambda_{b, i} / \lambda_{e, i}$ in a decreasing order, i.e., $\lambda_{b, 1} / \lambda_{e, 1}>\lambda_{b, 2} / \lambda_{e, 2}>\cdots>\lambda_{b, N} / \lambda_{e, N}$. Then, comparing the value of $\lambda_{k}$ and $\lambda_{b, N} / \lambda_{e, N}$, if $\lambda_{e, N} / \lambda_{b, N}<$ $\lambda_{k}<\lambda_{b, N} / \lambda_{e, N}$ is satisfied, $\lambda_{e, 1} / \lambda_{b, 1}<\lambda_{k}<\lambda_{b, 1} / \lambda_{e, 1}$ is also satisfied. If not, we will continue to compare the value of $\lambda_{k}$ and $\lambda_{b, N-1} / \lambda_{e, N-1}$ until the appropriate $n_{\text {inact }}$ is obtained. The steps of this algorithm are summarized in Algorithm 2.

It is noteworthy that two conditions should be satisfied to ensure the existence of the solution. Firstly, $\lambda_{e, i} / \lambda_{b, i} \leq$ $\lambda_{b, i} / \lambda_{e, i}$ should be satisfied to ensure that the interval $\left(\lambda_{e i} / \lambda_{b i}, \lambda_{b i} / \lambda_{e i}\right)$ is nonempty. Otherwise, we will change the strategy to maximize the MSE of the $k$ th transmitter to Eve link. Secondly, $\varepsilon_{k}$ should be set to a reasonable value to make sure that the right-hand side in (35) is negative and the solution exists. Accordingly, following condition should be satisfied:

$$
\begin{gathered}
\sqrt{\frac{\lambda_{b, i}}{\lambda_{e, i}}} \sum_{i=1} \frac{\sqrt{\lambda_{b, i} \lambda_{e, i}}}{\lambda_{e, i}-\lambda_{b, i}}-\sum_{i=1}^{n_{a c t}} \frac{\lambda_{b, i}}{\lambda_{e, i}-\lambda_{b, i}}<\varepsilon_{k}-n_{\text {inact }} \\
\quad<\sqrt{\frac{\lambda_{e, i}}{\lambda_{b, i}} \sum_{i=1}^{n_{a c t}} \frac{\sqrt{\lambda_{b, i} \lambda_{e, i}}}{\lambda_{e, i}-\lambda_{b, i}}-\sum_{i=1}^{n_{a c t}} \frac{\lambda_{b, i}}{\lambda_{e, i}-\lambda_{b, i}}}
\end{gathered}
$$




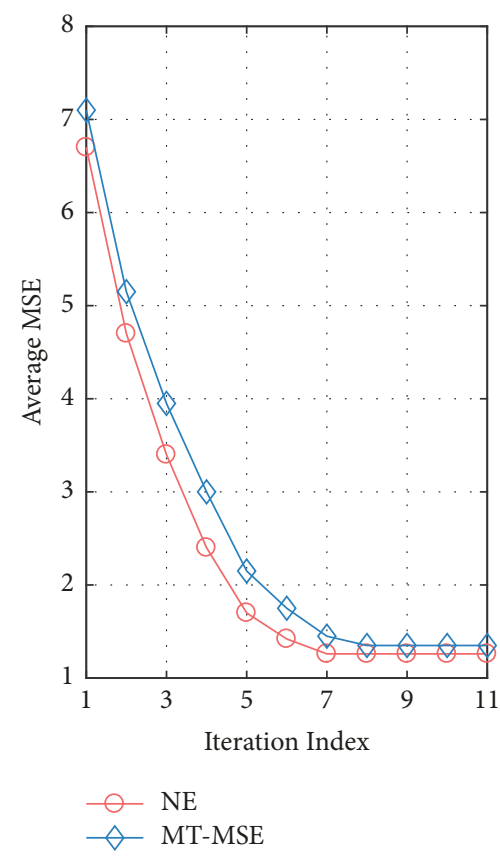

(a) $K=2$

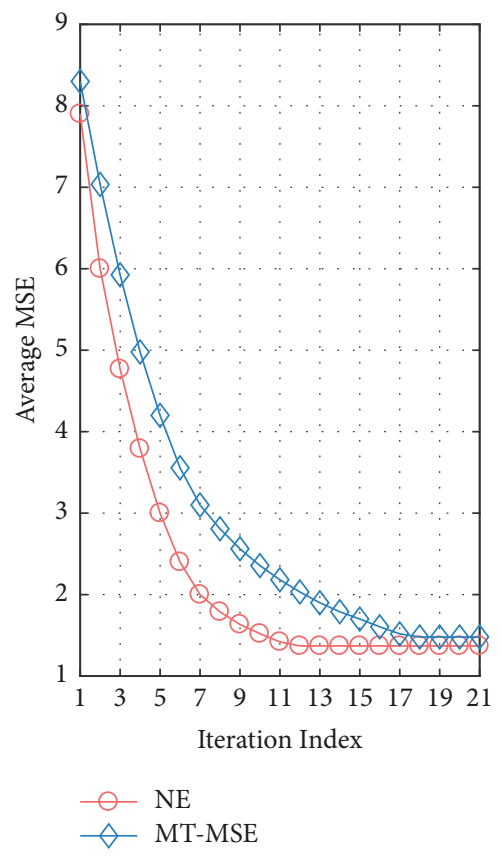

(b) $K=3$

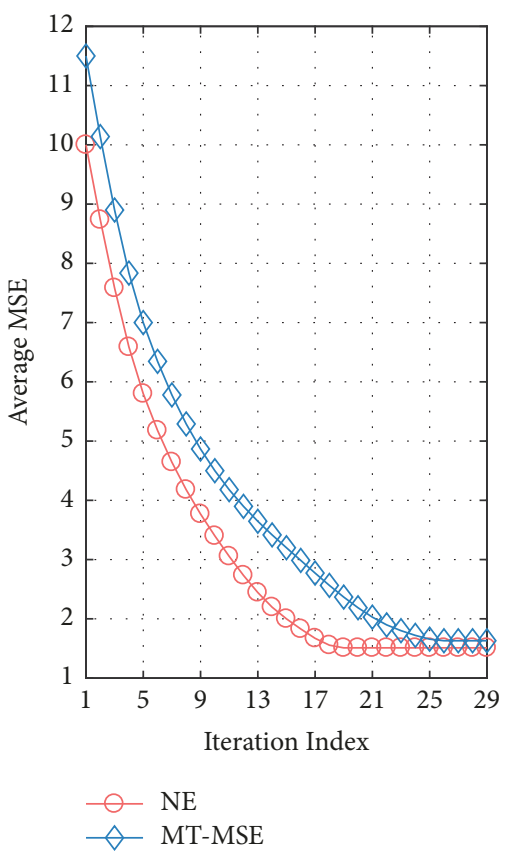

(c) $K=4$

FigURE 2: MSE performance of the 1st transmitter to the 1st receiver link.

TABLE 1: Iteration and CPU time of NE and MT-MSE algorithm.

\begin{tabular}{lcccc}
\hline & number of transmitter-receiver pairs & 2 & 3 & 4 \\
\hline \multirow{2}{*}{ NE } & iteration & 7 & 12 & 19 \\
& CPU time (s) & 16.17 & 38.88 & 82.08 \\
& average CPU time (s) & 2.31 & 3.24 & 4.32 \\
\hline \multirow{2}{*}{ MT-MSE } & iteration & 8 & 18 \\
& CPU time (s) & 26.16 & 92.88 & 26 \\
& average CPU time (s) & 3.27 & 5.16 & 8.41 \\
\hline
\end{tabular}

\section{Simulation Analysis}

In this section, simulation experiments are conducted to evaluate the performance of the proposed algorithm. Specially, we consider a MIMO interference network with $K$ legitimate transmitter-receiver pairs, and any receiver could be the potential Eve. In the network, both transmitters and receivers are equipped three antennas. The elements of the estimated channel matrices $\overline{\mathbf{H}}_{l k}$ and $\overline{\mathbf{H}}_{l e}$ are assumed to be i.i.d. zero-mean unit-variance complex-valued Gaussian random variables with variances $\xi_{\mathbf{H}_{l k}}=\xi_{\mathbf{H}_{l}}=0.01$. The power of background noise is assumed to be the same for all transmitter-receiver pairs and Eve, i.e., $\delta_{k}^{2}=\delta_{e}^{2}=1$. For each scenario below, we randomly generate 500 channel realizations and any conclusion is the arithmetic mean of 500 trials.

5.1. Convergence Performance. In the network we considered, there is no difference among the legitimate transmitterreceiver pairs statistically. Therefore, without any loss of generality, the performance of the 1st transmitter-receiver pair is selected as a representative of other transmitter-receiver pairs. We set the MSE constraint to 2, i.e., $\varepsilon_{1}=\varepsilon_{2}=\cdots=$ $\varepsilon_{k}=2, \forall k \in\{1,2, \ldots, K\}$, and generate the joint transceivers accordingly. The networks contain 2,3 , and 4 transmitterreceiver pairs are simulated, respectively, and the convergence behaviors of proposed algorithm based on NE are shown in Figure 2. As a comparison, we also use the Minimum Total MSE (MT-MSE) algorithm in [17] which pursues global optimality to solve the joint transceivers. We observe that final MSE values of two algorithms are convergent over iterations. Moreover, NE algorithm converges to a slightly smaller MSE as compared to MT-MSE algorithm. Since the result is based on statistic, all transmitter-receiver pairs could be considered to have similar channels, so the NE solution and the global solution are very close. But in some extreme cases in which channels of transmitter-receiver pairs are quite different, the two algorithms will lead to very different solutions and it is hard to tell which is better. However, there is no doubt that NE algorithm has a faster convergence, especially in networks with more transmitter-receiver pairs. As shown in Table 1, we observe that MT-MSE algorithm takes more CPU time in each iteration and is rapidly increased with the number 


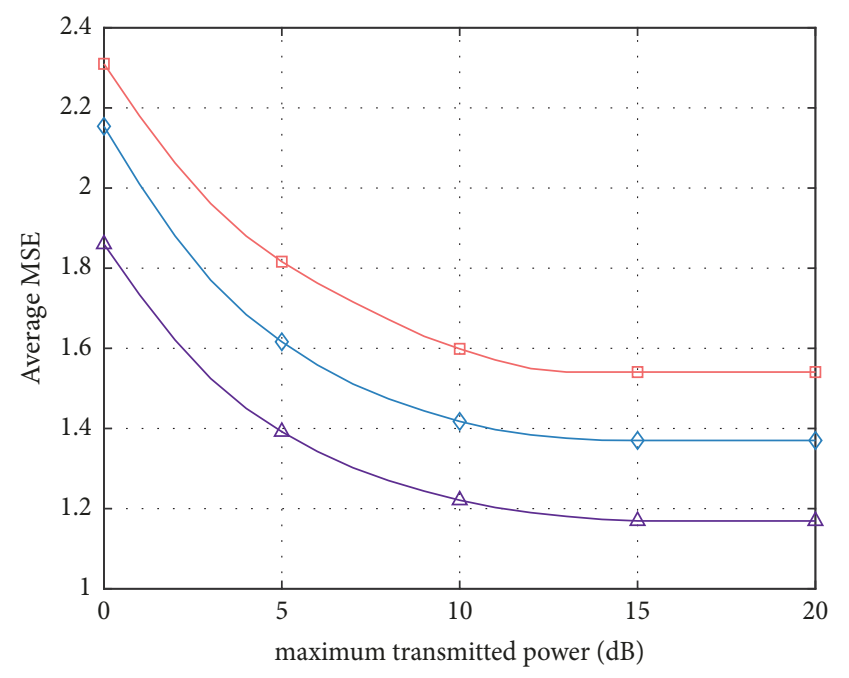

$\begin{array}{ll}\square & m=4 \\ \triangleright & m=3 \\ \triangle & m=2\end{array}$

FIGURE 3: MSE performance of the 1st transmitter to the 1st receiver link.

of transmitter-receiver pairs increasing. Conversely, for NE algorithm, average CPU time in each iteration is increased slightly. This can be explained by the fact that we obtain closed-form solution in each iteration of NE algorithm while only an approximately optimal solution can be obtained in MT-MSE algorithm. In each iteration, NE algorithm leads to more precise optimal solution while the complexity is relatively small. This also explains why NE algorithm has higher convergence speed.

5.2. Security Performance. The MSE and secrecy rate performance in networks of the number from 2 to 4 transmitterreceiver pairs are simulated. As shown in Figure 3, the MSE of the 1st transmitter to the 1st receiver link decreases with the transmitted power increasing, and the convergence values are smaller than the MSE of the 1st transmitter to Eve link (the minimum threshold is set to 2). It means that the communication quality of the 1st transmitter-receiver pair is better than Eve. Moreover, we note that the MSE of the 1st transmitter to the 1st receiver link is larger than 2 when transmitted power is small. In such case, the transmitted power is insufficient, which causes the constraint in (30) to be unsatisfied and the optimal TPC matrix fails to be achieved. Therefore, to obtain the best MSE performance, enough transmitted power must be provided in the actual application. In addition, the mutual interference becomes more serious as the number of transmitter-receiver pairs grows, which leads to the increase of MSE.

In PLS, secure communications can be guaranteed when the secrecy rate is positive. The secrecy rate performance of networks with 3 transmitter-receiver pairs is shown in Figure 4 . The secrecy rate without PLS coding is zero, which means that the 1st transmitter-receiver pair has no advantage in physical layer comparing to Eve. On the contrary, under

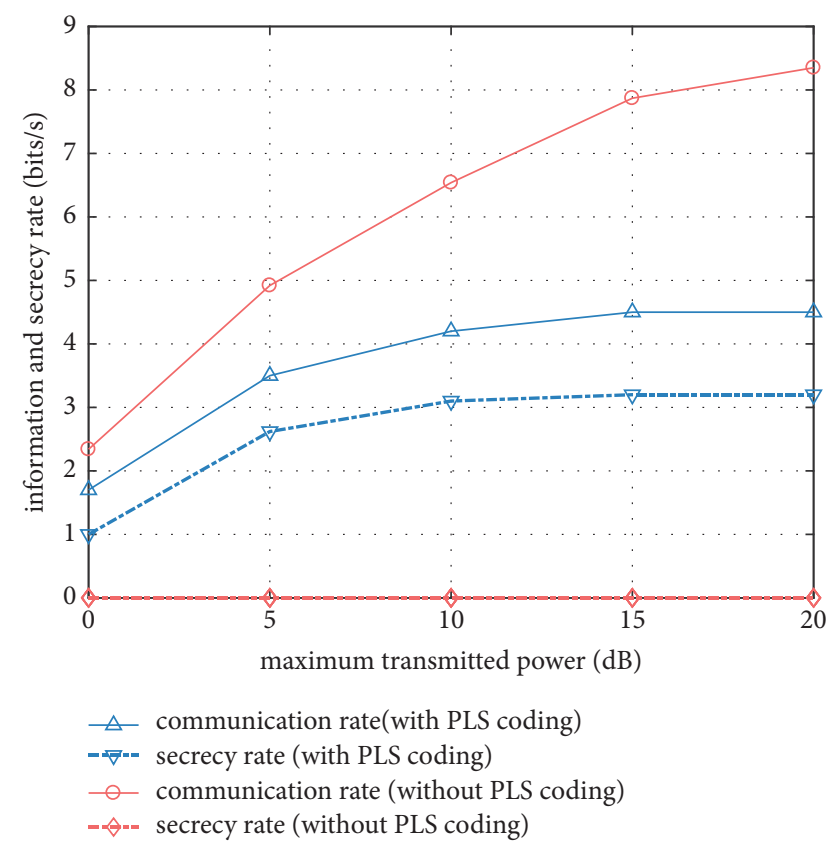

FIGURE 4: Secrecy performance of the 1st transmitter to the 1st receiver link.

effect of PLS coding, the secrecy rate becomes positive and is proportional to transmitted power, which means the 1st transmitter-receiver pair can defend against eavesdropping effectively. However, the information rate is lower under applying PLS coding. This can be explained by the fact that the PLS coding sacrifices a certain fraction of attainable communication rate in order to obtain a useful positive secrecy rate of data transmission. In other words, our strategy is a compromise between information rate and security performance. Moreover, the secrecy rate stops growing when the maximum transmitted power constraint is over $14 \mathrm{~dB}$. As mentioned in Section 4, required power corresponds one-toone to the MSE constraint $\varepsilon_{k}$. Therefore, once $\varepsilon_{k}$ is fixed, the ideal power of optimal transceiver is determined and higher transmitted power will not improve the secrecy performance anymore.

\section{Conclusion}

In this paper, we investigated secure communications for a MIMO interference network with $K$ transmitter-receiver pairs. A security strategy based on maximizing the difference of MSE between legitimate transmitter-receiver pairs and Eve is proposed. Specifically, the MSE of the signals between the legitimate transmitter-receiver pairs is minimized while the MSE of the signals decoded by Eve is kept higher than a certain threshold. The MSE minimization problem is formulated as a noncooperative game, and the NE solution is obtained by an asynchronous algorithm. In each iteration, the calculation of TPC matrices is sufficiently decoupled and the close-formed optimal solution is obtained. As a benefit, the computational complexity is highly reduced. The simulation results demonstrated that our security strategies 
can provide a positive secrecy rate and protect the network from eavesdropping. The proposed asynchronous algorithm is also confirmed to have faster convergence speed and shorter calculation time compared to iterative algorithm pursuing the global optimality.

\section{Appendix}

\section{A. Proof of the Existence of Nash Equilibrium}

In [25], Nash demonstrated the existence of equilibrium points in $\mathrm{n}$-person games by Kakutani's fixed-point theorem.

Theorem A.1 (Kakutani's fixed-point theorem). Given $\mathrm{X} \subseteq$ $\mathbb{R}^{n}$, let $\mathrm{S}(x): \mathrm{X} \ni x \longrightarrow \mathrm{S}(x) \subseteq \mathrm{X}$ be a multifunction. Suppose that the following hold:

(a) $\mathrm{X}$ is a nonempty, compact, and convex set;

(b) $\mathrm{S}(x)$ is a convex-valued correspondence and has a closed graph.

Then, there exists a fixed point of $\mathrm{S}(x)$.

For classical n-person games, assumption (b) of Theorem A.1 can be simplified into two sufficient conditions [26] and [27].

Theorem A.2 (existence of NE). Consider a strategic form $\mathscr{G}=\left(\mathscr{K},\left\{Q_{k}\right\}_{k \in \mathscr{K}},\left\{\mathcal{U}_{k}\right\}_{k \in \mathscr{K}}\right)$, where $\mathscr{K}$ is a finite set. Suppose that

(a) Each $Q_{k}$ is a nonempty, compact, and convex subset of a finite-dimensional Euclidean space;

(b) One of the two following conditions holds:

(1) Each utility function $\mathcal{U}_{k}\left(\mathbf{T}_{k}, \mathbf{T}_{-k}\right)$ is continuous on $Q$ and, for any given $\mathbf{T}_{-k} \in Q_{-k}$, it is quasiconcave on $Q_{k}$;

(2) Each utility function $\mathcal{U}_{k}\left(\mathbf{T}_{k}, \mathbf{T}_{-k}\right)$ is continuous on $\mathbb{Q}$ and, for any given $\mathbf{T}_{-k} \in \mathbb{Q}_{-k}$, the optimization problem

$$
\max \underset{\mathbf{T}_{k} \in \mathbb{Q}_{k}}{\mathcal{U}_{k}}\left(\mathbf{T}_{k}, \mathbf{T}_{-k}\right)
$$

admits a unique optimal solution.

Then, game $\mathscr{G}$ admits a pure-strategy NE.

Here, the proof of existence of NE algorithm we proposed is given as follows.

Proof. According to (25), the TPC matrix of the $k$ th transmitter is transformed into the product of a constant matrix and a diagonal matrix. Due to the power constraint, coefficients of the diagonal matrix are $\varphi_{i} \in\left[0, p_{i}\right], \forall i \in\{1,2, \ldots, N\}$, which means that $Q_{k}$ is bounded. Moreover, $Q_{k}$ is a nonempty, compact, and convex subset of a $\mathrm{N}$-dimensional Euclidean space. Thus, Theorem A.2 (a) is satisfied.

According to (27), the utility function $\mathcal{U}_{k}\left(\mathbf{T}_{k}, \mathbf{T}_{-k}\right)$ is continuous and convex. And the closed-form solution of the

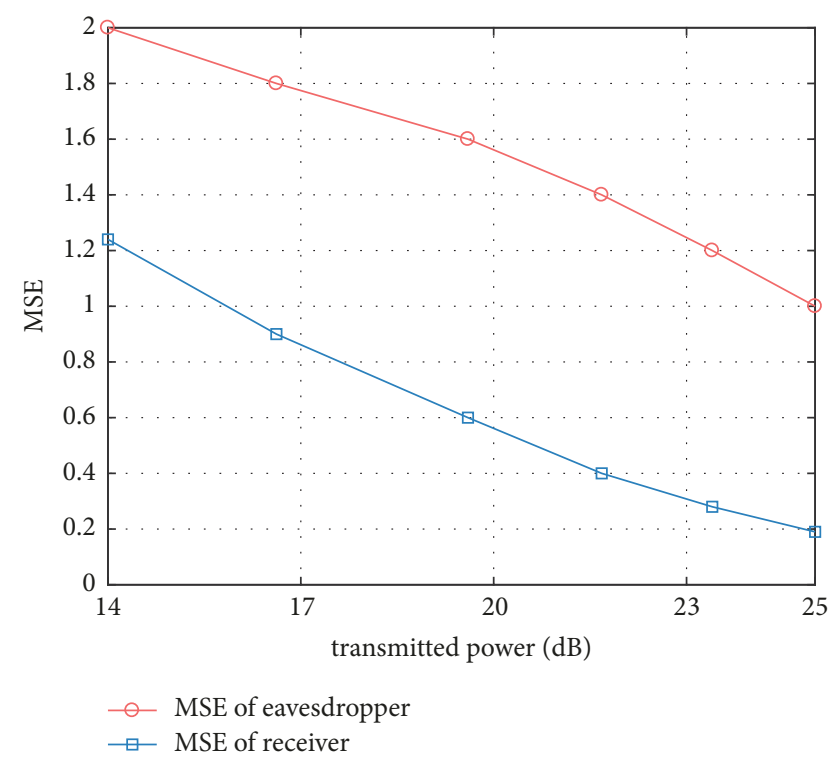

FIGURE 5: MSE versus transmitted power.

optimization problem (27) is given by (34), which means that the solution is unique and optimal. Therefore, Theorem A.2 (b.2) is satisfied. And the existence of NE is proved.

\section{B. Proof of the Inverse Correlation between MSE and Transmitted Power}

Proposition B.1. The minimum threshold of power constraint $p_{k \text {,min }}$ is inversely proportional to the MSE constraint $\varepsilon_{k}$.

Proof. According to (35), we have $\varepsilon_{k}=\sqrt{1 / \lambda_{k}} \alpha-\beta+$ $n_{\text {inact }}$, where $\alpha=\sum_{i=1}^{n_{\text {act }}}\left(\sqrt{\lambda_{b, i} \lambda_{e, i}} /\left(\lambda_{e, i}-\lambda_{b, i}\right)\right)<0, \beta=$ $\sum_{i=1}^{n_{a c t}}\left(\lambda_{b, i} /\left(\lambda_{e, i}-\lambda_{b, i}\right)\right)<0$, and $\lambda_{k}>0$.

Taking the derivative of it with respect to $\lambda_{k}$ into consideration, we have $d \varepsilon_{k} / d \lambda_{k}=-(1 / 2) \alpha \lambda_{k}{ }^{-3 / 2}>0$, which means that $\varepsilon_{k}$ is in direct proportion to $\lambda_{k}$.

Taking the derivative of (34) with respect to $\lambda_{k}$ into consideration, we have $d \sigma_{i} / d \lambda_{k}=-(1 / 2) \sqrt{\lambda_{b, i} / \lambda \lambda_{e, i}}\left(\lambda_{b, i}-\right.$ $\left.\lambda_{e, i}\right) /\left(\lambda_{b, i}-\lambda_{e, i} \sqrt{\lambda_{b, i} / \lambda \lambda_{e, i}}\right)^{2}<0$, which means that $\sigma_{i}$ is in inversely proportion to $\lambda_{k}$.

In conclusion, $\sigma_{i}$ is in inversely proportion to $\varepsilon_{k}$ while $p_{k \text {, min }}=\sum_{i}^{n_{\text {act }}} \sigma_{i}$; thus $p_{k \text {,min }}$ is in inversely proportional to the security constraint $\varepsilon_{k}$.

A MIMO PLC network consists of three transmitterreceiver pairs and an Eve is selected to verify this proposition. We set $\varepsilon_{k}$ from 1 to 2 , and the optimal joint transceiver of the 1st transmitter-receiver pair is generated as described in Section 4. As shown in Figure 5, the transmitted power is increased with $\varepsilon_{k}$ decreasing. This can be explained by the fact that when the transmitted power increases, the SNR will increase accordingly. And both the legitimate receiver and the eavesdropper will get better evaluations of data symbol, which cause the decrease of MSE. Therefore, there is 


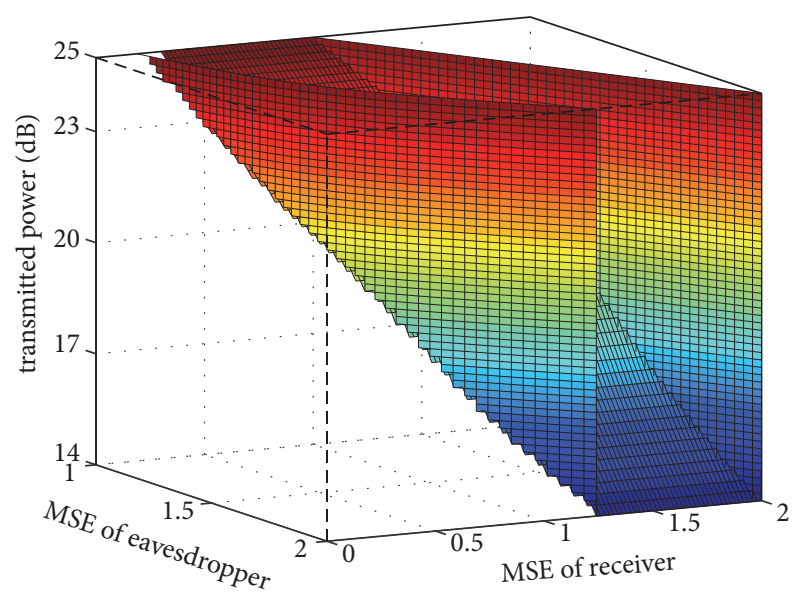

FIGURE 6: Solution space of the optimization problem.

a one-to-one correspondence between minimum threshold of transmitted power $p_{k \text {,min }}$ and MSE constraint $\varepsilon_{k}$. In other words, once $\varepsilon_{m}$ is set, any value of transmitted power higher than $p_{k \text {,min }}$ will not get any extra benefit in MSE. For clarity, the solution space of $\left\{\operatorname{tr}\left(\mathbf{M S E}_{\mathrm{Tx}_{k}, \text { Eve }}\right), \operatorname{tr}\left(\mathbf{M S E}_{\mathrm{Tx}_{k}, \mathrm{Rx}_{k}}\right), p_{k}\right\}$ is shown in Figure 6 . The solution space is a closed set in interval $\{[1,2],[0,2],[14,25]\}$. And the solutions in this set are not optimal except the points on the bottom face. In other words, there are varying degrees of transmitted power waste at any point above the bottom surface. And only the points on the left edge surface are the optimal solution of $\operatorname{tr}\left(\mathbf{M S E}_{\mathrm{Tx}_{k}, \mathrm{Rx}_{k}}\right)$. From the perspective of interference channel, when benefit of increasing transmitted power is not greater than the influence of the interference it caused, there is no need to raise the power anymore.

\section{Data Availability}

All data included in this study are available upon request by contact with the corresponding author.

\section{Conflicts of Interest}

The authors declare that they have no conflicts of interest.

\section{References}

[1] D. Maamari, D. Tuninetti, and N. Devroye, "Multi-user Cognitive Interference Channels: A Survey and New Capacity Results," IEEE Transactions on Cognitive Communications Networking, vol. 1, no. 1, pp. 29-44, 2015.

[2] H. Yang, W. Shin, and J. Lee, "Linear Degrees of Freedom for K-user MISO Interference Channels With Blind Interference Alignment," IEEE Transactions on Wireless Communications, vol. 16, no. 3, pp. 1921-1934, 2017.

[3] J. Nam, "Capacity bounds for the $K$-user Gaussian interference channel," Institute of Electrical and Electronics Engineers Transactions on Information Theory, vol. 63, no. 10, pp. 6416-6439, 2017.

[4] M. Benammar, P. Piantanida, and S. Shamai, "Capacity results for the multicast cognitive interference channel," Institute of
Electrical and Electronics Engineers Transactions on Information Theory, vol. 63, no. 7, pp. 4119-4136, 2017.

[5] M.-M. Zhao, Y. Cai, Q. Shi, M. Hong, and B. Champagne, "Joint Transceiver Designs for Full-Duplex K-Pair MIMO Interference Channel with SWIPT," IEEE Transactions on Communications, vol. 65, no. 2, pp. 890-905, 2017.

[6] W. K. Harrison, J. Almeida, M. R. Bloch, S. W. McLaughlin, and J. Barros, "Coding for secrecy: An overview of errorcontrol coding techniques for physical-layer security," IEEE Signal Processing Magazine, vol. 30, no. 5, pp. 41-50, 2013.

[7] M. Bloch, M. Hayashi, and A. Thangaraj, "Error-Control Coding for Physical-Layer Secrecy," Proceedings of the IEEE, vol. 103, no. 10, pp. 1725-1746, 2015.

[8] A. Mukherjee, S. A. A. Fakoorian, J. Huang, and A. L. Swindlehurst, "Principles of physical layer security in multiuser wireless networks: a survey," IEEE Communications Surveys \& Tutorials, vol. 16, no. 3, pp. 1550-1573, 2014.

[9] X. Chen, D. W. K. Ng, W. H. Gerstacker, and H.-H. Chen, "A Survey on Multiple-Antenna Techniques for Physical Layer Security," IEEE Communications Surveys \& Tutorials, vol. 19, no. 2, pp. 1027-1053, 2017.

[10] Q. Li, W.-K. Ma, and D. Han, "Sum Secrecy Rate Maximization for Full-Duplex Two-Way Relay Networks Using AlamoutiBased Rank-Two Beamforming," IEEE Journal of Selected Topics in Signal Processing, vol. 10, no. 8, pp. 1359-1374, 2016.

[11] L. Jiang, H. Tian, C. Qin, S. Gjessing, and Y. Zhang, "Secure Beamforming in Wireless-Powered Cooperative Cognitive Radio Networks," IEEE Communications Letters, vol. 20, no. 3, pp. 522-525, 2016.

[12] X. Gong, H. Long, H. Yin, F. Dong, and B. Ren, "Robust amplify-and-forward relay beamforming for security with mean square error constraint," IET Communications, vol. 9, no. 8, pp. 1081-1087, 2015.

[13] Q. Shi, W. Xu, J. Wu, E. Song, and Y. Wang, "Secure beamforming for MIMO broadcasting with wireless information and power transfer," IEEE Transactions on Wireless Communications, vol. 14, no. 5, pp. 2841-2853, 2015.

[14] L. Li, C. Huang, and Z. Chen, "Cooperative Secrecy Beamforming in Wiretap Interference Channels," IEEE Signal Processing Letters, vol. 22, no. 12, pp. 2435-2439, 2015.

[15] A. Kalantari, S. Maleki, G. Zheng, S. Chatzinotas, and B. Ottersten, "Joint power control in wiretap interference channels," IEEE Transactions on Wireless Communications, vol. 14, no. 7, pp. 3810-3823, 2015.

[16] S. A. Fakoorian and A. L. Swindlehurst, "Competing for secrecy in the MISO interference channel," IEEE Transactions on Signal Processing, vol. 61, no. 1, pp. 170-181, 2013.

[17] Z. Kong, S. Yang, F. Wu, S. Peng, L. Zhong, and L. Hanzo, "Iterative Distributed Minimum Total MSE Approach for Secure Communications in MIMO Interference Channels," IEEE Transactions on Information Forensics and Security, vol. 11, no. 3, pp. 594-608, 2016.

[18] C. Zhong, T. Ratnarajah, Z. Zhang, K.-K. Wong, and M. Sellathurai, "Performance of rayleigh-product MIMO channels with linear receivers," IEEE Transactions on Wireless Communications, vol. 13, no. 4, pp. 2270-2281, 2014.

[19] M. Razaviyayn, M. Sanjabi, and Z.-Q. Luo, "Linear transceiver design for interference alignment: complexity and computation," Institute of Electrical and Electronics Engineers Transactions on Information Theory, vol. 58, no. 5, pp. 2896-2910, 2012. 
[20] R. Feng, Q. Li, Q. Zhang, and J. Qin, "Robust secure beamforming in MISO full-duplex two-way secure communications," IEEE Transactions on Vehicular Technology, vol. 65, no. 1, pp. 408-414, 2016.

[21] H. Jing and A. L. Swindlehurst, "Robust secure transmission in MISO channels based on worst-case optimization," IEEE Transactions on Signal Processing, vol. 60, no. 4, pp. 1696-1707, 2012.

[22] C. E. Shannon, "Communication theory of secrecy systems," Bell Labs Technical Journal, vol. 28, no. 4, pp. 656-715, 1949.

[23] R. W. Newcomb, "On the simultaneous diagonalization of two semi-definite matrices," Quarterly of Applied Mathematics, vol. 19, pp. 144-146, 1961/1962.

[24] M. R. D. Rodrigues and P. D. M. Almeida, "Filter Design with Secrecy Constraints: The Degraded Parallel Gaussian Wiretap Channel," in Proceedings of the IEEE GLOBECOM 2008 2008 IEEE Global Telecommunications Conference, pp. 1-5, New Orleans, LA, USA, November 2008.

[25] J. Nash, "Equilibrium points in $N$-person games," Proceedings of the National Acadamy of Sciences of the United States of America, vol. 36, pp. 48-49, 1950.

[26] J. B. Rosen, "Existence and uniqueness of equilibrium points for concave N-person games," Econometrica, vol. 33, no. 3, pp. 520 $534,1965$.

[27] G. Scutari, D. P. Palomar, and S. Barbarossa, "Competitive optimization of cognitive radio MIMO systems via game theory," in Proceedings of the International Conference on Game Theory for Networks, Istanbul, Turkey, 2009. 


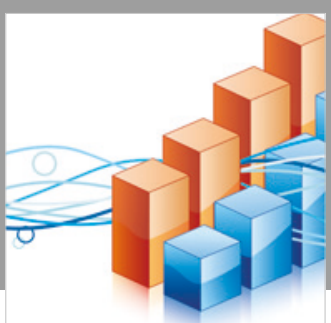

Advances in

Operations Research

\section{-n-m}
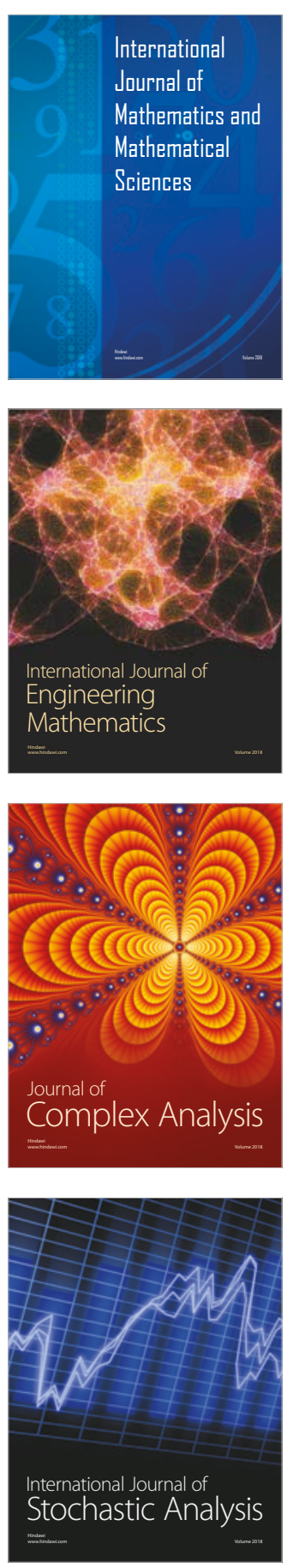
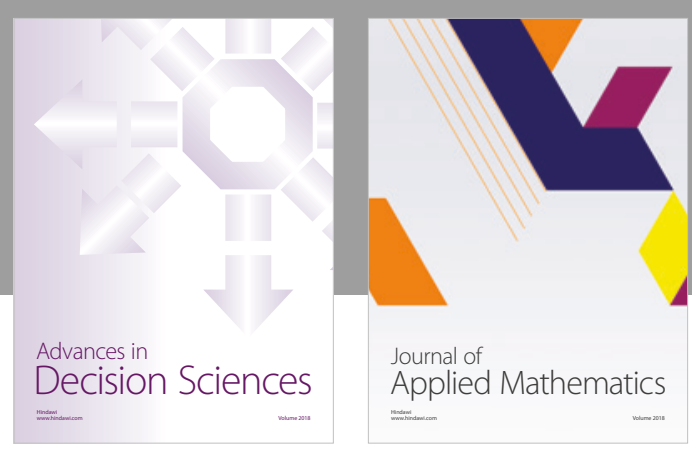

Journal of

Applied Mathematics
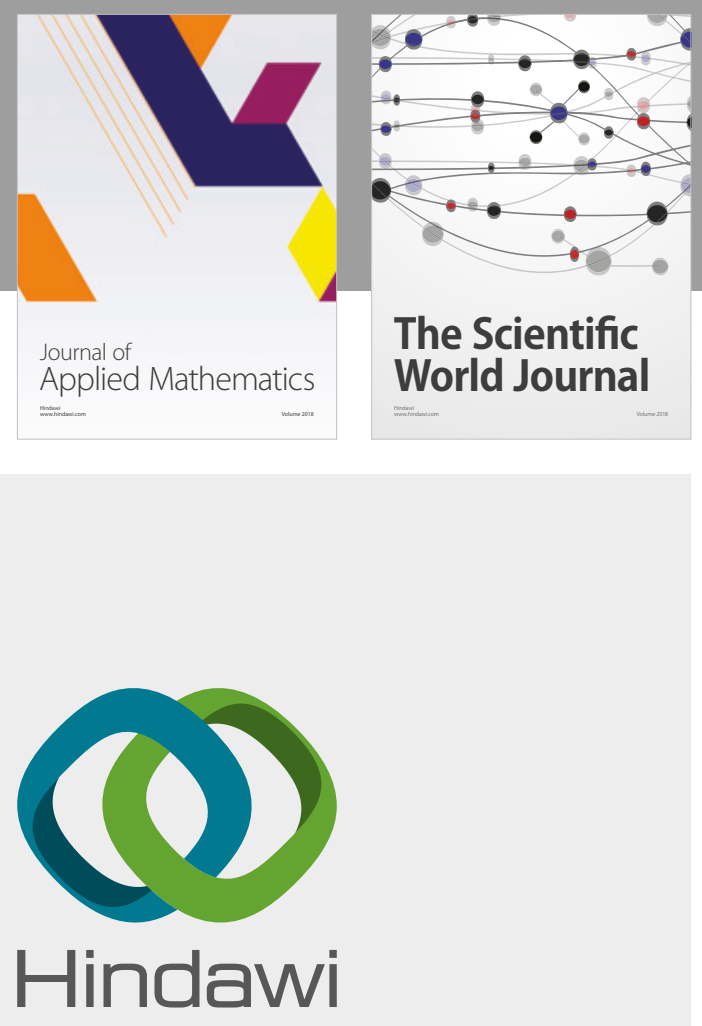

Submit your manuscripts at

www.hindawi.com

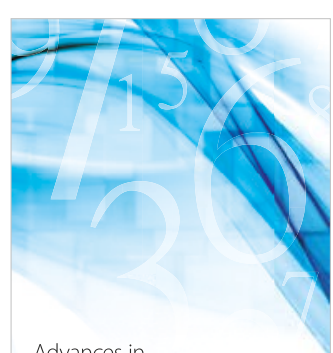

Advances in
Numerical Analysis
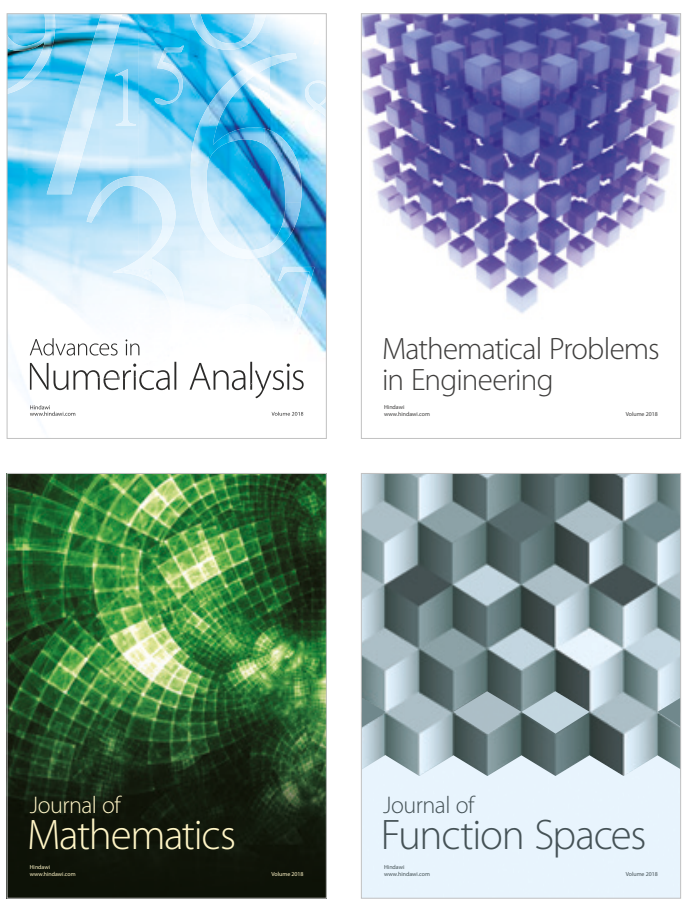

Mathematical Problems in Engineering

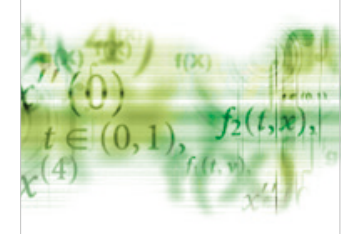

International Journal of

Differential Equations

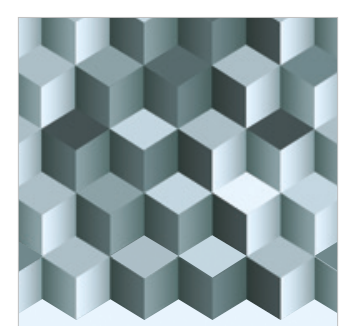

Journal of

Function Spaces

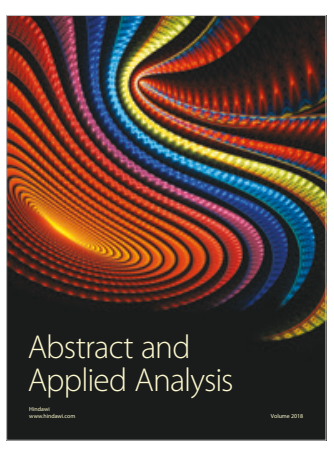

The Scientific

World Journal

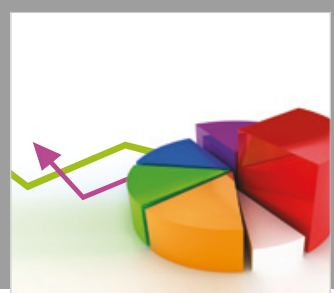

Journal of

Probability and Statistics
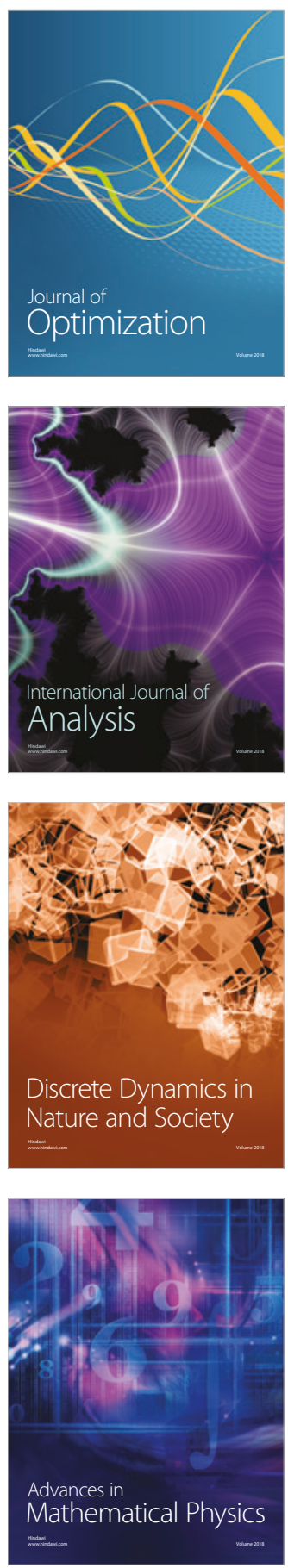